Indian J. Anim. Hlth. (2020), 59(1) : 11-16

DOI: 10.36062/ijah.59.1.2020.11-16

\title{
AFRICAN SWINE FEVER: AN EMERGING THREAT TO PIG HUSBANDRY IN INDIA
}

\author{
S. KUMAR ${ }^{1}$, T. K. DUTTA ${ }^{* 1}$, P. ROYCHOUDHURY ${ }^{1}$, H. DAS ${ }^{1}$, \\ F. AKTER ${ }^{1}$ AND P. K. SUBUDHI ${ }^{1}$
}

${ }^{1}$ Department of Veterinary Microbiology, College of Veterinary Sciences and Animal Husbandry, Central Agricultural University, Selesih, Aizawl-796 014, Mizoram, India

\begin{abstract}
African swine fever (ASF) is a highly contagious and deadly viral disease spreading rapidly in more than 50 pig rearing counties in the world. Warthog and other wild suids act as a reservoir host of the virus and the disease can transmit via direct contact or indirectly through vectors or fomites. Soft ticks under the genus Orinthodoros acts as a biological vector, but it can also be mechanically transmitted through Stomoxys calcitrans fly. First outbreak of ASF had been reported from China during January, 2019. Since emergence of ASF outbreak has also been reported in North Eastern Region of India, adjoining to China during April, 2020, the possible threat to pig husbandry in North Eastern States of India is discussed in this article.
\end{abstract}

Key words: African swine fever, Assam, Epidemiology, India

Globally Pork is considered to be the most consumed meat $(37 \%)$ followed by chicken (35.2\%) and beef $(21.6 \%)$ (FAO, 2013). In the present scenario pork has become a crucial protein food replacer due to fast growth, efficient feed conversion, quick turnover, and prolificacy of pig. The pork industry is considered to have the potential to meet the increasing demand of animal protein at a cheaper price compare to other meat. In India, although the piggery sector is growing at a steady pace, but it is confined to some specific regions of the country. According to the $20^{\text {th }}$ Livestock census, Assam ranked first in pig population although a decline of $12.03 \%$ population was observed over the previous livestock census. The decline in pig population may be due to lack of commercial pig farms, marketing chain, good policies related to pig farming etc., but the primary factor for economic losses in pig husbandry was due to various diseases like classical swine fever, porcine reproductive and respiratory syndrome (PRRS) etc. from time to time. In recent past, another exotic disease, ASF was being reported from NE Region (www.oie.int), which has significantly high morbidity and mortality in pigs leading to economic consequences associated with production losses.

African swine fever (ASF) is a highly contagious and deadly disease of pigs. ASF was first described in Africa in the 1920s (Costard et al., 2009). According to OIE, the mortality rate of ASF may be as high as $100 \%$. ASF is a notifiable disease to the World Organisation for Animal Health (OIE). ASF started its journey from Africa and so far, the disease has reached in more than 50 countries (Fenollar and Mediannikov, 2018)

*Corresponding Author 
around the world. The spreading of virus from endemic area to naïve area is facilitated by both legal and illegal movements of live animals, as well as the importation of animal products, byproducts, and animal feed (Brown and Bevins, 2018). As recently, ASF has reported in the North eastern region of India, the possible way of prevention and control of the dreadly disease in Indian context is discussed in this article.

\section{Epidemiology of ASF}

ASF was initially reported from East Africa in 1921 and the first outbreak was reported in Portugal in 1957 (Sánchez Vizcaíno et al., 2012). Subsequently, during 1970 and 1980, ASF emerged in several parts Europe (Netherlands, Italy, France and Belgium) and America (Cuba, the Dominican Republic, Haiti and Brazil) (Brown and Bevins, 2018). In the year 2007, major outbreaks had been reported from many countries viz.Georgia, Armenia, Azerbaijan and the European parts of Russia, Ukraine and Belarus. In Asian continent, the first ASF outbreak was reported from China in August 2018 followed by Mongolia (January 2019), Vietnam (Februray 2019), Cambodia (March 2019), North Korea (May 2019), HonKong (May 2019) and Mayanmar (September 2019).

Recently, India has also been reported the first case of ASF in the North eastern region adjoining to China (https://www.oie.int/wahis_2/public/ wahid.php/Reviewreport/Review? page_refer $=$ MapFull Event Report \& reportid = 34283). Subsequently aproximately 2500 pigs were reported dead within 15-20 days period from different regions of Assam and Arunachal Pradesh (www.pig-world.co.uk) and the representative specimens from dead pigs of Assam and Arunachal Pradesh were tested positive for ASF by National Institute of High Security Animal Diseases (NIHSAD, Official Communication No. 15-34/20NIHSAD dated 29/04/2020), Bhopal, India. As per literatures, Warthogs (Phacochoerus africanus) were considered to be the original vertebrate host of ASF and the soft tick Ornithodoros moubata was acting as intermediate host for completing the sylvatic life cycle (Jori et al., 2013). This vertebrate host was considered the most important asymptomatic reservoir of ASFV in Africa, besides waterhogs, bushpigs and red river hogs were found to be transmitted ASF virus to other pigs but they were not considered to be important reservoir (Jori and Bastos, 2009; Ravaomanana et al., 2011). ASFV can also infect other wild suids, but their role in the epidemiology of the disease is negligible (Jori and Bastos, 2009; Mur et al., 2012).

ASFV could be transmitted to healthy pigs either through direct contact with infected pigs (domestic or wild) or indirect contact with contaminated fomites, vector, and feeding contaminated pork products (Mazur-Panasiuk et al., 2019). Airborne transmission was also observed under laboratory setting, where animals were densely housed (Lichoti et al., 2017). In Africa, O. moubata ticks were major source of infection with ASFV for both domestic and wild pigs (Sánchez-Vizcaíno et al., 2019). Transmission of ASFV in O. moubata ticks through transstadial, transovarial and sexual, allows the persistence of ASFV infection even in the absence of definitive hosts (Frant et al., 2017). Apart from Ornithodoros, no other hematophagous invertebrate has been identified as a biological vector for ASFV, but mechanical transmission via Stomoxys calcitrans has been reported within $48 \mathrm{~h}$ of a blood meal on an infected pig (Olesen et al., 2020). The ASFV may also spread through transportation of infected live pigs and pork products. The ASFV persists in nature under different cycles viz. sylvatic cycle (wild pig - tick), domestic cycle (pig-pig) and wild boar cycle (Olesen et al., 2020).

\section{Characteristics of ASF virus}

ASF virus is the lone organism belongs to genus Asfivirus under the family Asfaviridae. The virus contains a dsDNA as genome with a genome length of 170-190 kbp depending on the isolate. The genome encodes between 160 and 170 genes and has covalently closed ends and terminal 
inverted repeats. An irregular lipoprotein envelope derived from plasma membrane gives the virion an irregular spherical shape measuring about $200-220 \mathrm{~nm}$ in diameter. The icosahedral capsid consists of 1892 or 2172 capsomeres. The capsid in turn encloses a second lipoprotein membrane, which surrounds the DNA containing core. Although, this virus is the only member of family, restriction enzyme analysis of DNA of different isolates revealed five groups (clads). All American and European isolates fall in one group (clad), whereas African isolates exhibit significant variation probably due to long time circulation of virus in Africa. Twenty-three genotypes have been described based on the partial sequences of the $\mathrm{p}^{72}$ gene (Brown and Bevins, 2018). All 23 genotypes are present in Africa, whereas only genotypes I and II have been found to be present outside of the African continent. ASFV replicates in the cytoplasm of the mononuclear phagocytic cells. The endoplasmic reticulum plays a very important role in viral assembly and envelopment (RomeroBrey and Bartenschlage, 2016). ASFV is a very resilient virus and can survive for a longer period of time under different environmental conditions. Live ASFV could be detected in frozen meat even after 1000 days (FAO, 2013). Virus can sustain in different raw and processed materials including chilled, fried, smoked, offals, blood, skin etc ranging from 11 days to 1000 days (FAO, 2013).

\section{Clinical symptoms and post mortem lesions}

The warthogs and bushpigs generally acted as asymptomatic carrier and serve as a source of infection to susceptible pigs and ticks (sylvatic cycle) (Gallardo et al., 2017; Alvarez et al., 2019). Neonatal warthogs develop a asympomatic viremia which transmit the virus to new ticks (larvae, nymph and young adult) during their engorgement (Netherton et al., 2019). Soft ticks act as the only route of transmission between infected and susceptible warthogs (Golnar et al., 2019). Sexual transmission is not observed in warthogs, however, the ASFV virus is found in seminal secretions (Schulz et al., 2017). To date, there is no conclusive data suggesting a is carrier state; however, a survey conducted in central Kenya found ASFV in asymptomatic domestic swine and warthogs (Ståhl et al., 2019). Pigs of all the age groups equally susceptible (Brown and Bevins, 2018).

The incubation period varies from 4-19 days. Clinical disease occurs in four forms; peracute, acute, subacute, and chronic (Yoo et al., 2020). In peracute form, affected animals die without any visible clinical symptoms with mortality rate upto $100 \%$ (Gallardo et al., 2017). Acute infections are caused by highly virulent strains and are typically characterized by a high fever, anorexia, lethargy, weakness, recumbency, diarrhea and/or constipation, abdominal pain, hemorrhagic signs, respiratory distress, nasal and conjunctival discharge, and abortions in pregnant females. Death often occurs within 710 days after the onset of clinical signs. Moderately virulent strains result in subacute infection (often with high mortality in young animals and much lower mortality in older animals), where the clinical signs often include abortion, fever, and transient hemorrhaging with death or recovery occurring within 3-4 weeks (Sargsyan et al., 2018). Chronic infections are characterized by intermittent or low fever, loss of appetite, depression and in some instances, result in a fatal infection (Sargsyan et al., 2018). Domestic pigs are most infectious during the incubation period and may act as source of infection for two days before the presentation of clinical disease and recovered pigs may also act as source of infection for one month after the disappearance of clinical signs (FAO, 2013).

The lesions in acute ASF are similar to classical swine fever (CSF) or hog cholera and often difficult to differentiate on the basis of postmortem examination. The most consistent lesions include enlarged, friable and dark-red spleen, enlarged haemorrhagic gastro-hepatic and renal lymph nodes, petechial to ecchymotic haemorrhages in the kidney, oedema of gall bladder, interlobular oedema of lungs, petechial 
haemorrhages in the urinary bladder, visceral peritoneum and haemorrhages in lymph nodes of abdominal cavity and myocardium. The pigs infected with highly virulent strains of ASFV exhibit localized areas of reddening or cyanosis of the skin on the tips of ears, extremities of tail, legs and/or over the ham region. Moderately virulent strains also show similar lesions but enlarged spleen is not friable and appears normal in colour. In chronic form, the skin lesions are slightly raised and firm hyperaemic areas of $2 \mathrm{~cm}$ or more in diameter to areas of necrosis. Swollen joints and generalized lymphadenopathy are common observations. Pulmonary lobules show areas of consolidation with or without pleural adhesions.

Histological lesions in acute form of disease are seen in the antigen-processing cells of the lymphoreticular system, cortical and stromal areas of lymph nodes, red pulp of spleen and kuffer cells in liver sinusoids. Haemorrhages in the lymph nodes, kidney and heart are the most common findings. One of the most prominent features in chronic form of ASF is hyperplasia of lymphoreticular tissues.

\section{Diagnosis of ASF}

ASF could not be differentiated from classical swine fever (CSF) by either clinical or postmortem examinations. As the clinical symptoms and post mortem lesions were apperantely similar with CSF, laboratory techniques should be applied to differentiate the diseases and to provide any definitive diagnosis. In addition, bacterial septicaemias may also be considered for differential diagnosis.

Laboratory diagnosis must be done based on isolation and identification of the virus, detection of genomic DNA by polymerase chain reaction (PCR) or detection of tissue antigen by fluorescent antibody test (FAT). PCR is recognized as the most sensitive technique for detection of ASFV at a very early stage of infection in tissues, blood or sera. The OIE has validated and recommended three different PCR techniques based upon the previously published reports (Agüero et al., 2003; King et al., 2003; Fernández-Pinero et al., 2013).

In endemic areas, detection of ASF antibodies in pigs could be done through ELISA test, indirect fluorescent antibody (IFA) test, indirect immunoperoxidase test (IPT) are worth as no vaccine against ASFV is available so far (Laddomada et al., 2019).

Laboratory diagnosis of ASF is very difficult at field level due to lack of infrastructure, equipments, reagents, as well as delay in reaching the sample to the laboratory. The rapid pen-side test might be helpful in initial screening of the disease before confirmatory laboratory test.

\section{Prevention and Control}

Like most of the viral infections, no specific treatment is available against ASFV infection. In addition, to date, no vaccine is also available due to several key factors, including the lack of identification of protective antigens, incomplete understanding of virus-host cell interactions, and inadequate knowledge relative to the diversity of viral strains currently circulating in natural reservoirs (Sang et al., 2020). Many vaccine options have been tried with varying levels of success, including vaccines with naturally or experimentally deleted genes, subunit vaccines based on recombinant proteins, and DNA vaccines (Sang et al., 2020). Vaccine development for ASFV is ongoing and challenging due to the range of genetic and antigenic variability as well as the myriad of strategies utilized by the virus to evade the host's immune response. Further work is essential to develop a vaccine that is both safe and provides a high degree of protection across virulent ASFV strains (Alvarez et al., 2019).

The most appropriate method to control the disease is slaughter and proper carcass disposal of infected as well as suspected pigs followed 
by disinfection of contaminated area. Also, restricted movements of live pigs, their products from an infected area to non-infected area or vice-vera should be adopted as effective control measure. ASF is a vector-borne disease, so elimination of its vector is an important steps in controlling the disease. Care should also be taken for fomites (clothes, utensils, vehicles, shoes, etc.), which play an important role in spreading of infection. Strict biosecurity measures should be adopted in commercial as well as backyard farms to prevent the entry of infection in the noninfected areas. Swill feeding must be regulated by invoking stringent effort. Fences must be installed to block intruders such as roaming wild boar. Strict regulations and controls on food waste from international flights, and ships should be adopted at national level.

\section{Conclusion}

African swine fever (ASF) is a highly contagious and severe viral disease affecting domestic and wild pigs and considered as one of the most serious threat to pig husbandry globally. As the outbreak of ASF is reported from Assam and Arunachal Pradesh in India, it may pose a serious

\section{REFERENCES}

Agüero M, Fernández J, Romero L, Sanchez C, Arias M et al., 2003. Highly sensitive PCR assay for the routine diagnosis of African swine fever virus in clinical samples. J Clin Microbiol, 41: 4431-4434

Alvarez J, Bakker D and Bezos J, 2019. Epidemiology and control of notifiable animal diseases. Front Vet Sci, 6: 43

Brown VR and Bevins SN, 2018. A review of classical swine fever virus and routes of introduction into the United States and the potential for virus establishment. Front Vet Sci, 5: 31

Costard S, Wieland B, De Glanville W, Jori F, Rowlands $\mathrm{R}$ et al., 2009. African swine fever: how can global spread be prevented? Philos Trans R Soc Lond B Biol Sci, 364(1530): 2683-2696

FAO, 2013. Food Outlook Biannual Report on Global threat to the entire pig husbandry because of its extremely high level of morbidity and mortality. It is already indiacted that there is no specific treatment or protective vaccines are available against the disease. The disease transmission is also very stable through various animate and inanimate objects including ticks as biological and mechanical carrier. Therefore, utmost preacustions need to taken by the Governments to protect the pigs and the poor and marginal pig farmers of India to sustain the rural economy. Knowledge gained during the first entry and outbreak of PRRS in pig population in India in general and in the North eastern India in particular in 2011 should be applied in containment and control of the disease. Restriction of movement of animals and animal handlers including vehicles, feeds, processed pork products etc. from affected areas should strictly restricted by implementation of effective rules and regulations.

\section{ACKNOWLEDGEMENTS}

Authors thankful to the Dean of CVSc \& AH, CAU, Selesih, Aizawl, Mizoram-796014 for her support to complete this article.

Food Markets. ISSN: 0251-1959. http:// www.fao.org/3/a-I5703E

Fenollar F and Mediannikov O, 2018. Emerging infectious diseases in Africa in the 21st century. New Microbes New Infect, 26: 10-18

Fernández-Pinero J, Gallardo C, Elizalde M, Robles A, Gómez C et al., 2013. Molecular diagnosis of African swine fever by a new real†time PCR using universal probe library. Transbound Emerg Dis, 60(1): 48-58

Frant M, Wo Ÿniakowski G and Pejsak Z, 2017. African swine fever (ASF) and ticks. No risk of tickmediated ASF spread in Poland and Baltic states. J Vet Res, 61(4): 375-380

Gallardo C, Soler A, Nieto R, Cano C, Pelayo V et al., 2017. Experimental infection of domestic pigs with African swine fever virus Lithuania 2014 
genotype II field isolate. Transbound Emerg Dis, 64(1): 300-304

Golnar AJ, Martin E, Wormington JD, Kading RC, Teel PD et al., 2019. Reviewing the potential vectors and hosts of African swine fever virus transmission in the United States. Vector Borne Zoonotic Dis, 19(7): 512-524

Jori $\mathrm{F}$ and Bastos AD, 2009. Role of wild suids in the epidemiology of African swine fever. Ecohealth, 6(2): 296-310

Jori F, Vial L, Penrith ML, Pérez-Sánchez R, Etter E et al., 2013. Review of the sylvatic cycle of African swine fever in Sub-Saharan Africa and the Indian ocean. Virus Res, 173(1): 212-227

King DP, Reid SM, Hutchings GH, Grierson SS, Wilkinson PJ et al., 2003. Development of a TaqMan ${ }^{\circledR}$ PCR assay with internal amplification control for the detection of African swine fever virus. J Virol Methods, 107: 53-61

Laddomada A, Rolesu S, Loi F, Cappai S, Oggiano A et al., 2019. Surveillance and control of African swine fever in free-ranging pigs in Sardinia. Transbound Emerg Dis, 66(3): 1114-1119

Lichoti JK, Davies J, Maru Y, Kitala PM, Githigia SM et al., 2017. Pig traders' networks on the KenyaUganda border highlight potential for mitigation of African swine fever virus transmission and improved ASF disease risk management. Prev Vet Med, 140: 87-96

Mazur-Panasiuk N, Żmudzki J and Wożniakowski G, 2019. African swine fever virus-persistence in different environmental conditions and the possibility of its indirect transmission. $\mathrm{J}$ Vet Res, 63(3): 303

Mur L, Boadella M, Martinez-Lopez B, Gallardo C, Gortazar C et al., 2012. Monitoring of African swine fever in the wild boar population of the most recent endemic area of Spain. Transbound Emerg Dis, 59(6): 526-531

Netherton CL, Connell S, Benfield C and Dixon LK, 2019. The genetics of life and death: Virus-host interactions underpinning resistance to African swine fever, a viral haemorrhagic disease. Front Genet, 10: 402
Olesen AS, Belsham GJ, Bruun Rasmussen T, Lohse L, $\mathrm{B} ø \mathrm{dker} \mathrm{R}$ et al., 2020. Potential routes for indirect transmission of African swine fever virus into domestic pig herds. Transbound Emerg Dis, 00: $1-13$

Ravaomanana J, Jori F, Vial L, Perez-Sanchez R, Blanco E et al., 2011. Assessment of interactions between African swine fever virus, bushpigs (Potamochoerus larvatus), Ornithodoros ticks and domestic pigs in northwestern Madagascar. Transbound Emerg Dis, 58(3): 247-254

Romero-Brey I and Bartenschlager R, 2016. Endoplasmic reticulum: the favorite intracellular niche for viral replication and assembly. Viruses, 8(6): 160

Sánchez-Vizcaíno JM, Mur L and Martínez-López B, 2012. African swine fever: An epidemiological update. Transbound Emerging Dis, 59: 27-35

Sánchez-Vizcaíno JM, Laddomada A and Arias ML, 2019. African Swine Fever Virus. In Diseases of Swine (eds J.J. Zimmerman, L.A. Karriker, A. Ramirez, K.J. Schwartz, G.W. Stevenson and J. Zhang), ch 25, pp443-442

Sang H, Miller G, Lokhandwala S, Sangewar N, Waghela SD et al., 2020. Progress toward development of effective and safe african swine fever virus vaccines. Front Vet Sci, 7: 84

Sargsyan MA, Voskanyan HE, Karalova EM, Hakobyan LH and Karalyan ZA, 2018. Third wave of African swine fever infection in Armenia: Virus demonstrates the reduction of pathogenicity. Vet World, 11(1): 5-9

Schulz K, Staubach C and Blome S, 2017. African and classical swine fever: similarities, differences and epidemiological consequences. Vet Res, 48(1): 84

Ståhl K, Sternberg-Lewerin S, Blome S, Viltrop A, Penrith ML et al., 2019. Lack of evidence for long term carriers of African swine fever virusA systematic review. Virus Res, 272: 197725

Yoo D, Kim H, Lee JY and Yoo HS, 2020. African swine fever: Etiology, epidemiological status in Korea, and perspective on control. J Vet Sci, 21(2): e38

Received - 05.05.2020, Accepted - 25.05.2020, Published - 01.06.2020 